\title{
Research Article \\ Intelligent Government Control System Construction Based on 5G IoT
}

\author{
Xinyu Xu \\ College of Computing, University of Leeds, Leeds, West Yorkshire, UK \\ Correspondence should be addressed to Xinyu Xu; sc20xx@leeds.ac.uk
}

Received 13 October 2021; Revised 15 November 2021; Accepted 18 November 2021; Published 6 December 2021

Academic Editor: Guolong Shi

Copyright ( 2021 Xinyu Xu. This is an open access article distributed under the Creative Commons Attribution License, which permits unrestricted use, distribution, and reproduction in any medium, provided the original work is properly cited.

\begin{abstract}
This paper presents an in-depth analysis of the construction of an intelligent government control system in the context of 5G IoT. The research has conducted performance tests and network tests on the samples of the components which debugged the problems that occurred during the tests and analyzed and improved the system. The system designed in this study is not only easy to maintain but also very reliable and safe, with a high level of accuracy, and can be used for remote "five remote" and management of switchgear and other related power equipment. Based on this model, a dual middleware PaaS service selection algorithm is proposed, and a utility evaluation strategy is used to load balance the PaaS service. The test results show that the DMPS algorithm has a high selection accuracy and load balancing capability. Combined with the experience of local government investment and financing platform financing risk management and control practices, the financing risk control system established through the study is made more feasible. From the development history of local government investment and financing platforms, we study local government investment and financing platforms from the perspective of historical development and explore the root causes of their high financing risks and possible defects in the management process of daily work. Concerning the existing laws and regulations, practical operation norms, the framework of the local government investment and financing platform system, and other objective conditions, we start to build a financing risk control system for local government investment and financing platforms from both practical and operational management aspects, to find a financing risk control system that is in line with the regulations and effective. Finally, the system will be put into practice, and the effectiveness of the system will be tested by using the simulation operation method, and the results will be adjusted in time for the shortcomings of the system. The system is expected to help local government investment and financing platforms to reduce their risks so that they can provide better services for urban infrastructure construction and become a solid link in the economic development chain.
\end{abstract}

\section{Introduction}

$5 \mathrm{G}$ is based on $4 \mathrm{G}$, which is based on computer and information and communication technology. With the development of the mobile Internet, the scale of mobile communication capacity will grow further and rapidly in the future. This reality poses higher requirements and extremely serious challenges for network transmission. First, if the current conditions and network bandwidth only expanded, the capacity will not be able to meet the demand of the future high-speed development of traffic; both the bit cost and network consumption will be too large, even beyond the affordability [1]. Secondly, the rapid growth of network traffic in a short period will further increase the demand for spectrum resources (but the spectrum resources themselves are extremely scarce) and the current remaining available spectrum. The remaining spectrum resources are not only fragmented but also span a large area, so it is difficult to ensure the efficient use of all spectrum resources. Finally, the future development of mobile networks is bound to be characterized by heterogeneous and multiple coexistences. In short, to expand network capacity effectively, it is necessary to control and manage each network more efficiently and make the connection between networks easier, to give users a better experience. To address these issues and to better meet the future surge in mobile demand, new 5G 
mobile technologies have been introduced [2]. The establishment of a modular monitoring system for remote monitoring of switchgear based on 5G IoT technology is a development plan proposed in the context of the current trend of the development of power systems and technologies and information networks in China. This project uses advanced technologies such as 5G IoT, diverse sensing devices and related technologies, dynamic data collection and parameter measurement for power switches and the whole set of facilities and equipment, and advanced network technology, the data obtained by the sensing devices directly delivered to the remote application layer to realize various functions such as automatic protection, detection, and numerical measurement for switchgear. The system proposed in this study can meet the functions of remote dynamic monitoring of power switchgear, remote monitoring, and measurement of the parameters of power switchgear and can accurately and effectively grasp the actual situation of switchgear operation even if the staff is not at the switchgear operation site [3].

Smart government is one of the key elements of national institutional reform. Under the concept of smart government, the grassroots government is no longer just a service manager, but cogovernance with the masses to achieve value cocreation. It is necessary to set up a special inspection team and set up a financing risk specialist to inspect the process of each financing project. After the end of the financing process, summarize and analyze the effect of financing. To achieve intelligent governance at the grassroots level is to empower the power to operate under the sun through technological means [4]. On the one hand, the grassroots government uses intelligent means as a link to strengthen the top-level design. The grassroots government builds a multigovernance big data platform, changes the governance model, recreates intelligent means, increases efforts to improve grassroots governance services, enhances government administrative efficiency, and ultimately modernizes the level of national governance; on the other hand, the grassroots government uses new-generation information technology to measure and visualize the factors involved in public issues. Through the disclosure of data, the grassroots can grasp the information dynamics in a timely and accurate manner and gain full knowledge of the issues. Not only that, but it can also broaden and deepen the breadth and depth of the grass roots' understanding of the issues [5]. However, the current supply of public cultural services still suffers from a single supply body, insufficient supply initiation, regional supply imbalance, poor supply content, and supplydemand mismatch misalignment, making the strategic work of promoting and enhancing the development of the spiritual and cultural life of the whole society with quality public cultural services facing heavy difficulties. The concept of making full use of various emerging information technologies to effectively improve the level of urban public services and enhance urban management capacity can provide strong support and help to solve the many difficulties encountered in the current public cultural services, so the construction and development of smart cities provide the macroenvironment and conditions to improve the quality and quantity of public cultural service supply, and the concept, method, and technology of smart city construction can be used as the solution to the current public cultural services. Relying on technology as a systematic solution to many problems in the current public cultural service provision is an important topic worthy of attention in the field of public cultural service provision research.

However, because of historical legacy problems and some other objective environmental factors, their internal control is weak, their financing risk control is confused, and they lack the awareness of financing risk control, which makes their operation increasingly difficult. The purpose of this study is to make a breakthrough based on the original financing risk control methods of local government investment and financing platforms and to build a practical and more scientific risk control system. It provides a reference for strengthening financing risk control, enriching the methods of financing risk control, guaranteeing legal compliance, improving solvency, and reducing financing risks. Through the construction and implementation of the local government investment and financing platform financing risk control system to solve the existing problems, to improve the local government investment and financing platform financing risk control environment, improve efficiency, and reduce risk. Now the local government investment and financing platform are in a critical period of development, the overall indebtedness is high, and the financing of some local government investment and financing platforms often does not comply with the regulations, and the emergence of these situations has impacted this special platform struggling in the first line of urban infrastructure construction. Analyzing the root causes of financing risks arising from local government investment and financing platforms is more conducive to the construction of a risk prevention and control system. Therefore, this study has important implications for strengthening the risk control of local government investment and financing platform financing.

\section{Status of Research}

If debt risks occur during a period of rapid development of the country's economic level, they are likely to cause a slowdown in socioeconomic development. The rate of growth of local government debt and the rate of growth of the income of the local population determine whether debt risk will arise [6]. The risk of local government debt is due to the overexpansion of local governments due to lax control of their governmental debt. His research task local government debt risk can be divided into two types: the first is the risk of currency devaluation; the second is the credit risk of local governments, which can lead to a reduction in the credibility of local governments, both of which arise from the overexpansion of local governments due to the lack of strict control over their governmental debt [7]. The experiment uses the JMeter tool to send charging record data to the system at the same time using multiple threads to test the response time of the new system and the traditional system. The test results show that the traditional system uses direct storage, 
and the storage response time increases with the increase in the number of data storage. When the number of data storage exceeds 250 , the response time increases significantly, reaching $27.53 \mathrm{~s}$. The new system uses a task scheduling module. The main reason for the risk of local government debt is the decentralized behaviour of the government. He felt that the mismatch of governmental powers might trigger individual local governments to issue too many bonds, which would have a negative impression on their provision of services and construction of infrastructure for the public, and therefore he forced the government to fight for mutual correspondence of powers [8]. For its part, the government is more in dire need of a good bond market to ensure their level of fundraising. SOC analysis was applied to develop two basic models that can show the interest rate and the rate of return on capital to indicate what percentage of debt in net worth is required to achieve the best debt ratio. The empirical analysis and the calculation of a large amount of data were used to derive an upper bound for the optimal debt ratio and thus a risk warning signal.

With the development of IoT sterilization, some scholars have also researched service-oriented architecture (SOA) of IoT middleware. It proposed a service-oriented IoT software architecture, based on which an IoT service middleware was designed to realize the collaborative work between the sensing layer and the application layer through the centralized management of the entity services of the sensing layer and the application layer services and then provide the users with the unified invocation of IoT sensing services [9]. An IoT cloud sensor architecture is proposed based on Open Service Gateway Protocol (OSGI), and Atlas, a scalable IoT service middleware, is designed [10]. Atlas contains three parts: device driver, gateway communication, and cloud access, which can realize the dynamic access of different sensor objects [11]. An IoT middleware based on publish/subscribe mechanism is designed to realize the push and management of sensor-aware services. An SOA-based middleware for IoT monitoring systems is designed to solve the problem of fusion of heterogeneous monitoring data and provide users with service options for monitoring device sensing services [12]. The IoT middleware generally manages and selects services for the sense control services of IoT devices, and only the functional properties of the sense control services are considered in the service selection. In multi-PaaS IoT systems, the current middleware cannot consider the nonfunctional attributes of PaaS services and the actual demand preferences of end devices, resulting in poor performance of PaaS service selection [13].

The construction of a risk control system for financing local government investment and financing platforms. There are mainly related objectives, principles to be followed and methods to be used. To seek solutions to the problems that may exist in the current financing risk control of local government investment and financing platforms, the system is reshaped from both practical operation and operation management, and solutions are sought for the factors that may generate risks. The information software of the system and the digital terminal were debugged, and the data of the switchgear parameters obtained from the digital terminal were compared with the data obtained from the field test, and the sampling data obtained from the digital terminal were corrected; the communication protocol, networking mode, and control network of the system were adjusted and improved. Finally, the designed samples are verified and tested several times. The problems found in the test are analyzed and improved.

\section{Analysis of the Construction of the Intelligent Government Control System with 5G IoT}

3.1. Intelligent $5 G$ IoT Design. In recent years, with the development of computer technology specialization in different fields, some Internet companies have established a variety of PaaS platforms and are widely used in Internet systems; these PaaS platforms provide reliable, high-performance, and stable services, such as data visualization, video analysis, speech synthesis, and semantic parsing; these services can improve the system's intelligence level [14]. Applicationside middleware (ASM) simultaneously receives service response data from different PaaS platforms. The experiment selects the JMeter tool, uses different threads to simulate multiple PaaS platforms, and sends charging pile status data to ASM via HTTP. In addition, these PaaS platforms encapsulate the operating environment and functions, so the system does not need to develop the underlying services separately, but can be invoked directly, which greatly reduces the development cost and operation and maintenance cost of the system, and shortens the development cycle. With the popularization of 5G technology, high-bandwidth, and high-capacity mobile communication is widely used in IoT systems, making it a reality for IoT terminal devices and application layer software to call the PaaS platform for multiple specialized services. In the multi-PaaS IoT system, the data from the sensing layer terminal devices need to be processed by different PaaS platforms and then sent to the application layer, and the system network layer has a horizontal application architecture, which is different from the vertical application architecture of the traditional IoT system, making it difficult for the traditional middleware to meet the requirements. Currently, there are many PaaS platforms with different data access and data invocation methods, so the combination of IoT systems and multiple PaaS platforms has challenges such as data communication, service invocation, and service selection.

$$
\mathrm{HSM}_{\text {request }}=\left\{\mathrm{BM}_{\text {req }}, \mathrm{QM}_{\text {req, }}, \mathrm{QM}_{\text {prefer }}^{2}\right\} .
$$

With the rapid development of cloud computing technology, increasingly IoT PaaS platforms will be available, and the same type of service can be provided by multiple platforms. IoT systems allow access to multiple PaaS platforms at the same time, and the system needs to choose among many PaaS services with similar functions based on business requirements. Unlike the sensing services in traditional IoT systems, PaaS services have multiple operating parameters that change dynamically depending on the 
invocation situation. IoT end devices run at the edge of the system, and it is difficult to evaluate the parameters of different PaaS platforms and select the appropriate service at runtime due to the impact of computational performance and operational power consumption, etc. Therefore, in a multiPaaS IoT system, the middleware needs to choose accurately among many PaaS services with similar functions according to the actual needs of the system [15]. 5G technology's high bandwidth makes it possible to send a large amount of data from end devices to PaaS platforms for processing, and the number of devices accessing the system will increase, and the data that PaaS platforms need to process concurrently will continue to grow. The PaaS platform will pass the processed data into the application layer in bulk, which requires the application layer to have good access performance for high concurrency and a large volume of data incoming. Therefore, the middleware needs to be able to flexibly cope with a large amount of data access and achieve elastic adjustment of the load capacity to ensure system reliability, as shown in Figure 1.

In a multi-PaaS IoT system, the massive data uploaded by many end devices are first processed by PaaS services, and after the processing is completed, the ASM receives the service response data from multiple PaaS platforms. This has an impact on the performance of multiple PaaS platforms accessing ASM at the same time. Public welfare projects have long return periods, so the issuance of urban investment bonds for financing will generate higher financing risks.

$$
\begin{aligned}
s_{i} & =\left\{\begin{array}{l}
\text { baseModel }=\left(\mathrm{BP}_{1}, \mathrm{BP}_{2}, \cdots, \mathrm{BP}_{N}\right), \\
\text { QoSModel }=\left[T_{s}, T_{s}-T\right],
\end{array}\right. \\
\mathrm{QM}(t) & =\left\{f_{q 1}(t), \cdots, f_{q i}(t), \cdots, f_{q m}(t)\right\} .
\end{aligned}
$$

If the asynchronous approach is adopted, the data sending of the PaaS platform is decoupled from the logic of receiving and processing data at the ASM side, and the ASM responds immediately after receiving the network data and puts the data into the message queue before processing, which makes the data communication between multiple PaaS platforms and ASM noninterference and improves the communication efficiency between ASM and PaaS platforms. The end device access module of HSM mainly solves the problem of heterogeneous communication protocols of different end devices, which are connected to HSM through UART or Ethernet [16]. The terminal device access module is divided into three units: heterogeneous protocol reception, protocol parsing shared library, and data parsing. After the terminal device uploads the field data collected by the sensor, the original data is firstly received by the heterogeneous protocol receiving unit according to different communication methods, then the protocol parsing shared library unit finds the corresponding communication protocol template from the protocol type library according to the terminal device type, and finally the data parsing unit parses the original field data according to the communication proto- col parsing template to obtain the type and content of the field data.

After receiving raw data through the terminal access module, the HSM processes the raw data of the byte stream transmitted by the device through the data processing unit, which is divided into extraction, parsing, and normalization processes. When the data processing unit processes multiple raw data at the same time, the overall blocking cannot be caused by the time-consuming process of one raw data processing. In the data processing unit, HSM chooses a master-slave Reactor-based multithreaded approach for the different processes. The thread pooling technique creates a certain number of threads in the operating system before the processing task is executed, which are in the idle waiting queue before the task is assigned and are all in the idle waiting call state, these threads do not consume processor computing resources and occupy little memory space. When the data processing unit is assigned tasks such as extraction, parsing, and normalization, the thread pool assigns a free thread from the waiting queue to the task for processing. If too many tasks need to be processed at the same time and there are no free threads in the idle waiting queue, the thread pool creates new threads and when the data processing is completed, the HSM destroys some work threads that exceed a fixed number. Compared to creating/destroying work threads for each task, the data processing unit reduces system and resource overhead and improves system responsiveness by reusing multiple threads using thread pooling techniques, as shown in Figure 2. As a result, the financing risks of local government investment and financing platforms have further increased. To carry out large-scale financing, some local government investment and financing platforms will exaggerate the amount of project investment or forge the progress of the project to obtain more bank loans.

Data from multiple PaaS platforms will be sent to ASM at the same time, and ASM needs to respond to these data promptly. If time-consuming operations such as related storage or analysis are executed immediately after receiving the data, it will reduce the module's ability to handle multiple PaaS concurrently [17]. Task queues are implemented based on message queues, which can handle many tasks and ensure no data loss, and achieve decoupling of task reception and task execution so that the task scheduling module will execute reliably no matter how many tasks need to be processed. With the task scheduling scheme using the subscription publishing model, multiple task executors can subscribe to the task queue at the same time, so that the task execution capacity can be extended by increasing the number of task executors when many tasks need to be processed.

Service selection requests typically contain multiple requirement parameters, some of which must be satisfied and some of which need to be satisfied as much as possible. The service selection request analysis unit first models the different types of requirement parameters uniformly and then designs evaluation methods to satisfy the requirements separately. 


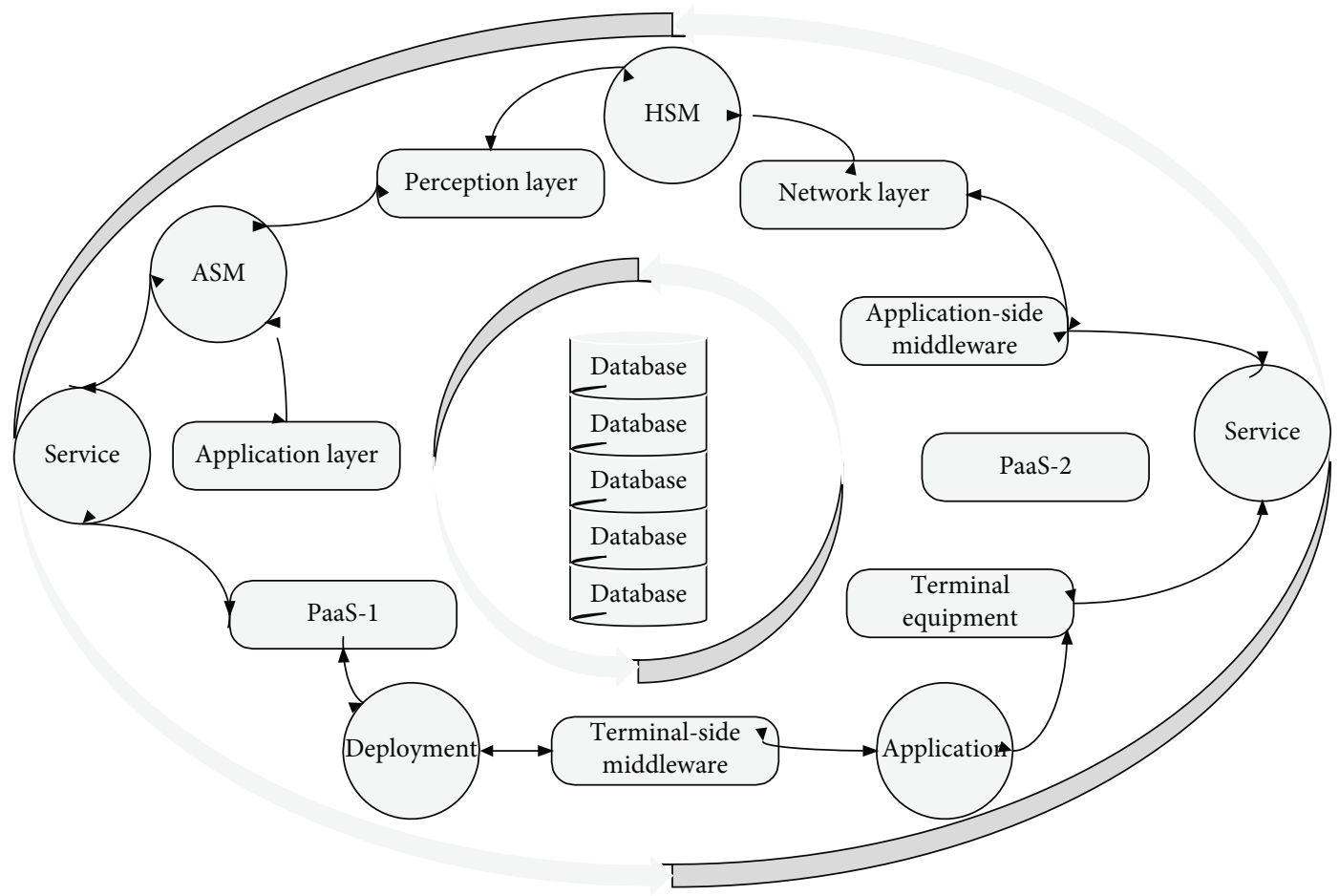

FIGURE 1: MPO-DM model.

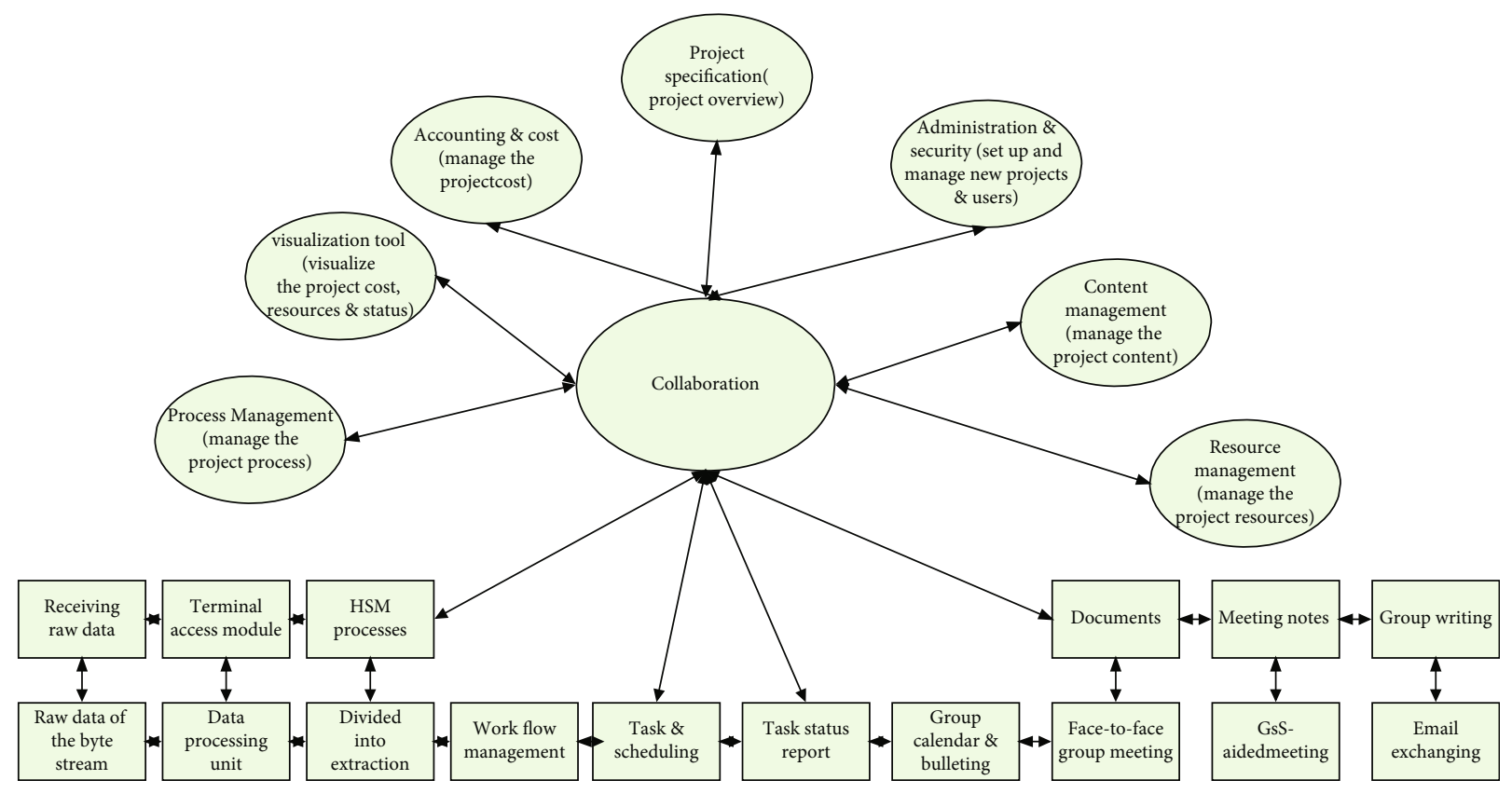

FIgure 2: Task scheduling module in ASM.

3.2. Intelligent Government Control System Construction. Then, the protocol analysis shared library unit finds the corresponding communication protocol template from the protocol type library according to the terminal device type, and finally the data analysis unit analyzes the original field data according to the communication protocol analysis template to obtain the type and content of the field data. Most of the local government investment and financing platforms currently do not have a sound early warning mechanism for financing risks, and it is difficult to achieve full process control of financing risks, and they can no longer predict risks in advance or detect them promptly, relying mainly on past financing experience to assess the scale and manner of future financing, combined with their profitability and 
solvency for risk assessment [18]. These steps can only be part of the financing risk early warning work. Because of the public welfare nature of local government investment and financing platforms, their projects are mostly urban infrastructure construction, with long cycles and poor profitability leading to their inability to have stable main business income and relatively stable profitability like other companies, and their business strategies and financing decisions often rely on the subjective judgment of their operations and managers, and they cannot make correct judgments about their operating conditions, profitability and development prospects. This brings greater risks to the financing of local government investment and financing platforms, coupled with the lack of a continuous early warning system for financing risks within local government investment and financing platforms, making the financing risks far beyond their affordability.

$$
\begin{aligned}
& U_{\text {prefer }}(j)= \begin{cases}\frac{q_{i j}^{\prime}}{r_{j}^{\prime}}, & q_{i j}^{\prime} \leq r_{j}^{\prime}, \\
0, & q_{i j}^{\prime}>r,\end{cases} \\
& U_{\text {entire }}=\sum_{j=1}^{k}\left(\frac{q_{i j}^{\prime}}{r_{j}^{\prime}}\right)^{2} .
\end{aligned}
$$

Because local government investment and financing platforms do not pay enough attention to the control of risk points in the financing process and at the same time the supervision of the use of financing funds is not in place, there are no planning and management of debt service funds, which has led to the further increase in financing risks of local government investment and financing platforms. Firstly, some local government investment and financing platforms will exaggerate the investment amount of projects or falsify the progress of projects to obtain more bank loans, or increase the scale of assets on their accounts by overcalculating business income, thus issuing more urban investment bonds, which makes their financing risks intensify. Secondly, individual local government investment and financing platform does not use the financing money according to the contract after obtaining the financing money, and misappropriates or even embezzles the financing money, making the financing money enter the highrisk areas or even personal hands, which will undoubtedly make the financing risk higher [19]. In a multi-PaaS IoT system, middleware needs to accurately choose among many PaaS services with similar functions according to the actual needs of the system. Finally, local government investment and financing platforms do not have effective methods for the management and use of debt service funds, and debt service funds are sometimes misappropriated or embezzled, thus bringing pressure on their repayment and generating debt service risks and even generating overdue debts, which further increases the financing risks.

$$
U(i)=\sum\left(\alpha_{j} \times U_{\text {entire }}(j)\right)-\beta \times U_{\text {prefer }}(j)
$$

Although the city investment Buddha that has been restructured into a corporate enterprise with independent legal person, but in the final analysis its establishment and survival are dependent on local government economic subsidies or land allocation, making it different from ordinary corporate enterprises, the actual power is mostly in the hands of the government, the management and even the board of directors' resolutions and strategies will be swayed by the government or even deviate from the market orientation, in terms of human resources management from employees. In terms of human resource management, from staff to senior management, there are cases of part-time appointment by local government officials or direct appointment by government management, and these managers are generally lacking in corporate management and risk judgment ability, unable to accurately grasp the market direction, and are prone to corporate management problems, making the operation and profitability of local government investment and financing platforms further decline, which also leads to the intensification of their financing risks, as shown in Figure 3.

The current profitability of the local government investment and financing platform is much lower than other enterprises, and the nonperforming asset rate is also rising year after year, catching up with the basic value of the industry all the way, regarding the control and management of financing risks ultimately hoped to use the five elements of internal control to reduce the cost of financing risks that may arise. And to improve the financing risk control system, make full use of the constraints and supervision functions between the various positions and departments, and finally achieve the goal of reducing financing risks. Through the construction of financing risk prevention and control system of local government investment and financing platform, it can correctly perceive its solvency, assess its profitability and asset structure to determine reasonable financing amount before financing, fulfil financing procedures following the law during financing, eliminate falsification, operate properly after financing, improve the overall quality of employees, guarantee the healthy and stable development of the enterprise, and be able to sustain profitability and ensure that debts can be repaid in time to strengthen the solvency of local government investment and financing platforms.

There are many ways to finance local government investment and financing platforms, such as bank loans, long and short-term loans, equity financing, etc., of which the issuance of corporate bonds is the most critical, to increase the scale of financing, local government investment and financing platforms often choose to issue debt financing. In the multi-PaaS IoT system, the data of the terminal device at the perception layer needs to be processed by different PaaS platforms and then sent to the application layer. The system network layer has a horizontal application architecture, which is compared with the vertical application architecture of the traditional IoT system. The big difference makes it difficult for traditional middleware to meet the requirements. The purpose of issuing urban investment bonds by local government investment and financing platforms is often to 

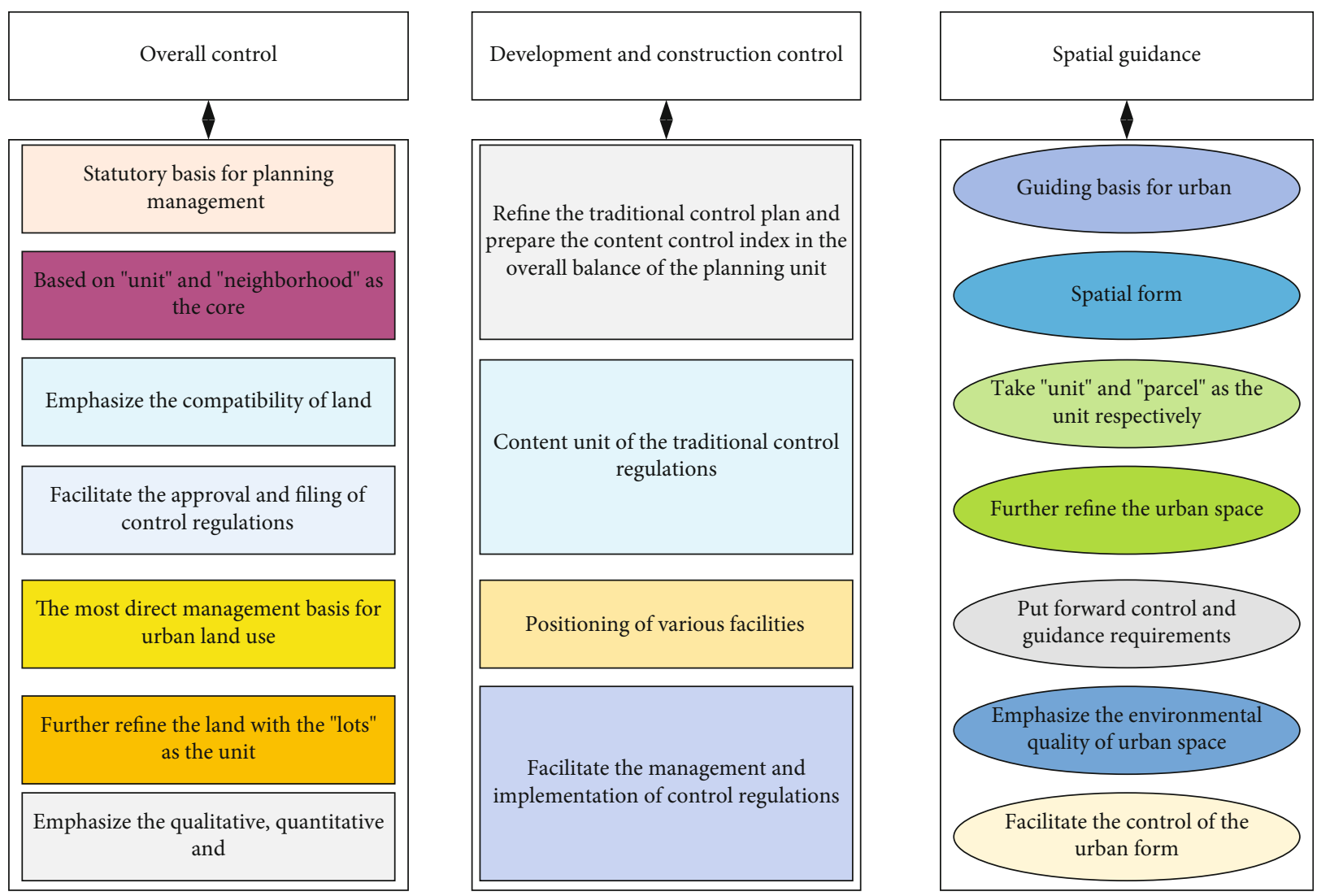

FIGURE 3: Planning and preparation system for the intelligent government control system.

carry out local urban infrastructure construction or some public welfare services and projects assigned by the local government, so the medium-term or long-term urban investment bonds issued will often be guaranteed by the government or the implicit guarantee of government credit to achieve the purpose of raising large amounts of funds. The urban investment bonds issued by local government investment and financing platforms are corporate bonds, which also adopt the process and way of issuing corporate bonds, from approval to project to repayment, the whole process is the same as ordinary corporate bonds, but the funds issued are used for public welfare projects with low return on investment and long return period, so the issuance of urban investment bonds for financing will generate higher financing risks. The following is an analysis of the financing process of local government investment and financing platforms, taking the issuance of urban investment bonds as an example. The overall process of issuing corporate bonds by local government investment and financing platforms are shown in Figure 4. From the clear financing demand to the final repayment of principal and interest, there are many risk points and human intervention in the whole process of issuing bonds, which will become the main source of financing risk for local government investment and financing platforms if not effectively controlled. The established financing risk control system can also be used for financing other types of financing after certain adjustments are made.
The grass roots, as an organic whole of the organization, operate with multiple actors. It can be summarized as three major actors: government, market, and individuals. It specifically contains four major actors: government, enterprises, social organizations, and the public. In grassroots governance, the main members of the government are the street offices and other departments where the grassroots are located. They are the most terminal governmental organizations as the government's dispatched agencies and must implement the governance instructions issued by the higher departments. The main members of enterprises are the various types of for-profit companies [20]. The core members of the people are the various types of organizations representing the people's demands and the individual people within the grassroots. To give full play to the organic dynamics of multiple actors in grassroots governance, we should establish a cooperative relationship led by the state, with the participation of enterprises, society, and the public, and with cooperation, mutual restraint, and a balanced situation. The problems and risks of cyberspace should be eliminated to the greatest extent possible through pluralistic participation, multidirectional interaction, and multiple systems. Therefore, the intelligent type must give full play to the collaborative effect of each governance subject, innovate to build an interactive platform for multiple subjects, strive to strengthen the open sharing of resources of multiple subjects, and build a convenient information feedback mechanism, so that the policy requirements and demands of each 


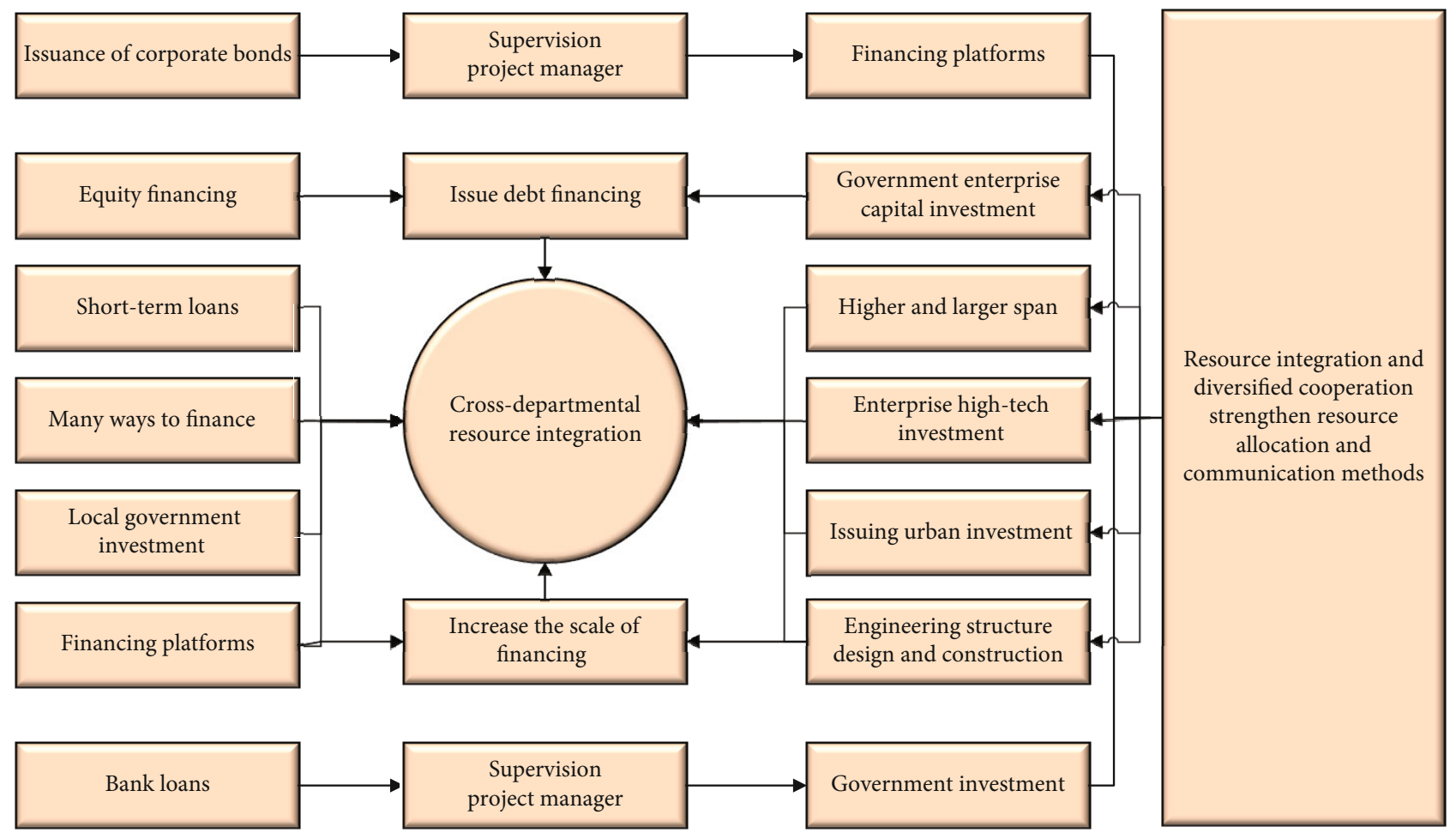

FIgURE 4: Resource integration and multicollaboration process.

subject can be reflected the grassroots government efficiently, thus enabling the government to better understand the relevant demands of each subject.

\section{Results and Analysis}

4.1. Smart 5G IoT Performance. With the development of IoT sterilization, some IoT middleware with a serviceoriented structure has emerged. This middleware centralizes the management of sensing services, and users can select the required sensing services through the middleware. The service selection capability provided by this IoT middleware is limited, and the selection is generally made by service keywords for user requirements, which is not very accurate. Therefore, it is a challenging challenge to select the accurate and appropriate services in IoT middleware. The application-side middleware (ASM) receives service response data from different PaaS platforms simultaneously. Therefore, the construction and development of smart cities provides a macroenvironment and conditional constraints for the supply of public cultural services. The concepts, methods, and technologies of smart city construction are used as solutions to the many problems existing in the supply of public cultural services. In systematic solution, the JMeter tool is selected for the experiment, and multiple PaaS platforms are simulated using different threads to send charging stake status data to the ASM in HTTP to test the communication parameters of the ASM receiving PaaS data: throughput rate, receive rate, send rate, and error rate (i.e., timeout failure rate), and the test results are shown in Figure 5.
The test results show that as the number of PaaS increases, the throughput rate, reception rate, and sending rate of the system increase, and the error rate is almost $0 \%$ when the number of $\mathrm{PaaS}$ is 400 and below but increases when the number of PaaS exceeds 500 due to the bottleneck in the performance of the single computer. In a practical scenario, the actual number of PaaS platforms that need to be accessed by the charging pile management system is much less than 400. Therefore, the IoT dual middleware-based charging pile management system with multiple PaaS access has high performance and can meet the actual demand. In the new system, ASM uses a task scheduling module to execute storage tasks, while the traditional system uses direct storage. To test the storage performance of the new system in a multi-PaaS environment, we compared the new system with the traditional system and designed experiments on the response time of stored data. The response time of the new system and the legacy system was tested by the JMeter tool using multiple threads to send charging records to the system at the same time, and the test results showed that the response time of the legacy system increased with the number of data storage using direct storage and the response time increased significantly to $27.53 \mathrm{~s}$ when the number of data storage exceeded 250 . In the task scheduling module, the response time remains stable and does not exceed $1.4 \mathrm{~s}$, and its storage performance is better than the traditional system, as shown in Figure 6.

The MPO-DM-based charging stake management system is designed and implemented. The system is designed in detail from three aspects: system architecture, system functions, and network topology, and the concrete 


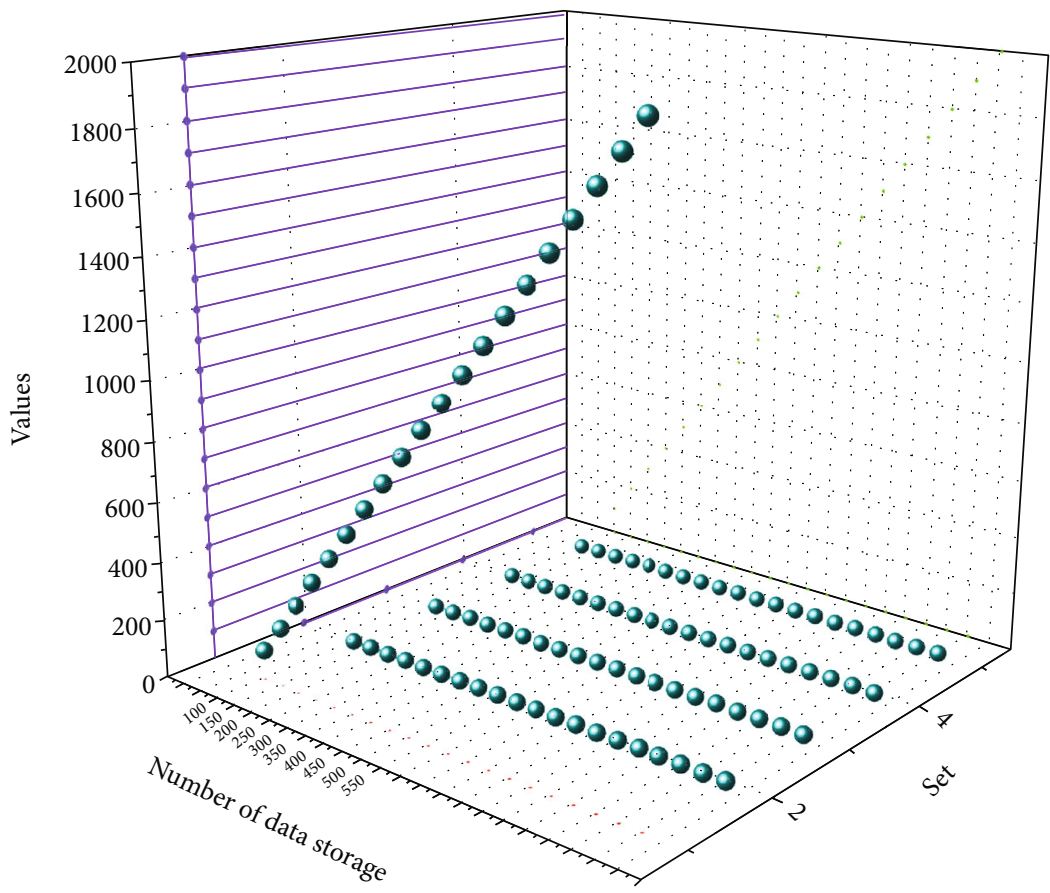

FIgURE 5: Multi-PaaS access performance test results.

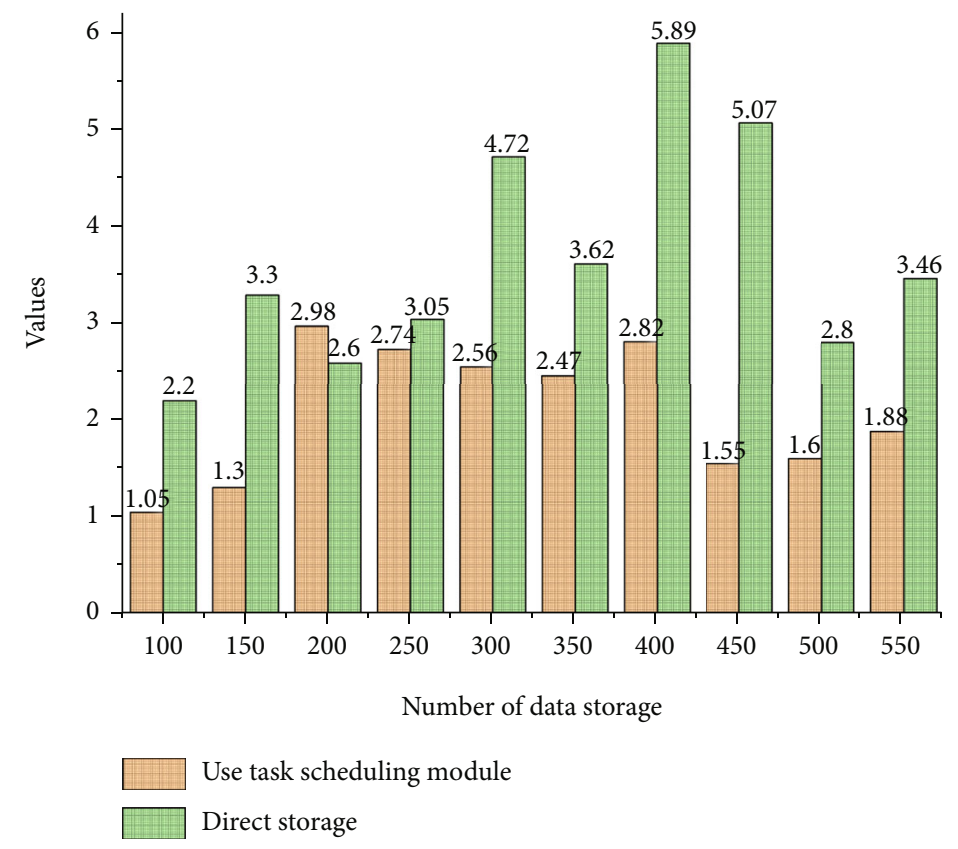

FIGURE 6: Storage response time test results.

implementation of terminal-side middleware and application-side middleware is given. Through the functional demonstration and performance test of the actual system, it shows that the system can interact with the PaaS platform and improve the data visualization level and user software convenience and has good multi-PaaS access and data storage performance, which effectively verifies the feasibility and effectiveness of the MPO-DM model. Realize various functions such as automatic protection, detection, and numerical measurement of the switch cabinet. The system proposed in this research can meet the requirements of remote dynamic monitoring of power switchgear, remote monitoring, and measurement of the parameters of the power switchgear; even if the staff is not at the operation site of the switchgear, they can accurately and effectively grasp the actual situation of the switchgear operation.

Based on the proposed dual middleware model of the multi-PaaS IoT system, the MPO-DM-based charging pile 
management system is designed and implemented, and the system is designed in detail from three aspects: system architecture, system function, and network topology, and the specific implementation is given for the end-side middleware and application-side middleware. Through the functional demonstration and performance test of the actual system, it shows that the system can interact with the PaaS platform and improve the data visualization level and user software convenience and has good multi-PaaS access and data response performance, which effectively verifies the feasibility and effectiveness of the MPO-DM model.

\section{Test Results of the Intelligent Government Control System}

The human resource management process constructed in this study was selected to adapt to the current situation of its part of the pilot application, combined with the actual human resource management situation, affirming that the recruitment process of professional knowledge and professional assessment is important for the subsequent talent management and talent pool but also helps to enhance the internal risk awareness of the enterprise, for the financing risk control having practical significance, to a certain extent, can play a role in reducing the financing risk. At the same time, the training methods mentioned in the framework have been tested in practice and have achieved good results, and the training on financing risk management has strengthened the risk response capability of internal staff and management. The training on financing risk management has strengthened the risk response-ability of internal staff and management, the training on corporate culture has strengthened corporate cohesion, the establishment of the assessment mechanism has standardized the behaviour of internal staff and improved work efficiency, and the professional competence of staff and team building has given more attention. In the future development and operation, we will strictly implement the appraisal system and continuously improve and amend the system to keep pace with the times and adapt to the strategic requirements of the enterprise. As a wholly-owned SOE under the local government, the salary system and reward and punishment system need to consider various factors, and many historical legacy issues are considered for the retirement benefits of employees. The new HRM framework has facilitated the development, improved the HRM process, enhanced the overall quality of internal staff, provided more professional talents for the control of financing risks, enhanced the competence of internal auditors, separated incompatible positions effectively, and formed a longterm mechanism for HRM optimization, as shown in Figure 7.

From the perspective of the environment of financing risk control, because the financing of local government investment and financing platform has government attributes with a government guarantee or potential guarantee of government credibility, whether applying for bank loans or issuing urban investment bonds are generally relatively smooth, banks and accounting firms will have a low default value for the financing risk of local government investment and financing platform, resulting in the management of local government investment and financing platform will spend a lot of time on financing. New 5G mobile technology is introduced. The establishment of a modular monitoring system for remote monitoring of switchgear based on $5 \mathrm{G}$ Internet of things technology is a development plan proposed under the background of the development trend of my country's power system and technology and information network. This has led the managers of local government investment and financing platforms to spend a lot of time on the use of financing funds while lacking corresponding attention to the financing itself. In addition, the local government investment and financing platform do not pay enough attention to the risk awareness training of the managers and the corresponding evaluation mechanism, which makes the managers of the local government investment and financing platform generally have a weak risk awareness and no motivation and ability to deal with financing risks. The use of local government investment and financing platform financing risk control systems can effectively enhance the risk awareness of internal managers.

The supervision and management of the finance department are crucial to the whole process of PPP project implementation. First, the finance department should strengthen supervision of the procurement process, especially in the selection of procurement methods, and strengthen supervision and inspection promptly, and take immediate measures to deal with violations once they are discovered. Second, the finance department should work with the implementation agencies of PPP projects to supervise and manage them well, and strictly prohibit borrowing government debts in the name of PPP projects to avoid transferring debts of social capital parties to local governments. Dilan establishes perfect government payment ledger information for PPP projects, includes government payment obligations in the government comprehensive financial report, and does not substitute current government purchase service expenditure for medium and long-term payment obligations of PPP projects to circumvent the evaluation procedures related to PPP projects, as shown in Figure 8.

As shown in Figure 8, the establishment and improvement of the financing risk control system of local government investment and financing platforms can enable local government investment and financing platforms to have rules to follow when carrying out financing activities. It can not only enhance the financing enthusiasm of local government investment and financing platforms but also restrain its financing behaviour and reduce the subjectivity and arbitrariness of local government investment and financing platforms. Therefore, it is necessary to control the key nodes that may generate financing risks in the financing process of local government investment and financing platforms, and try to refine and quantify the system standards. Therefore, it is difficult to ensure the efficient use of all spectrum resources. To effectively expand the network capacity, it is necessary to improve the utilization efficiency of network resources. At the same time, a special inspection team should be established and a financing risk commissioner should be set up to inspect the process of each 


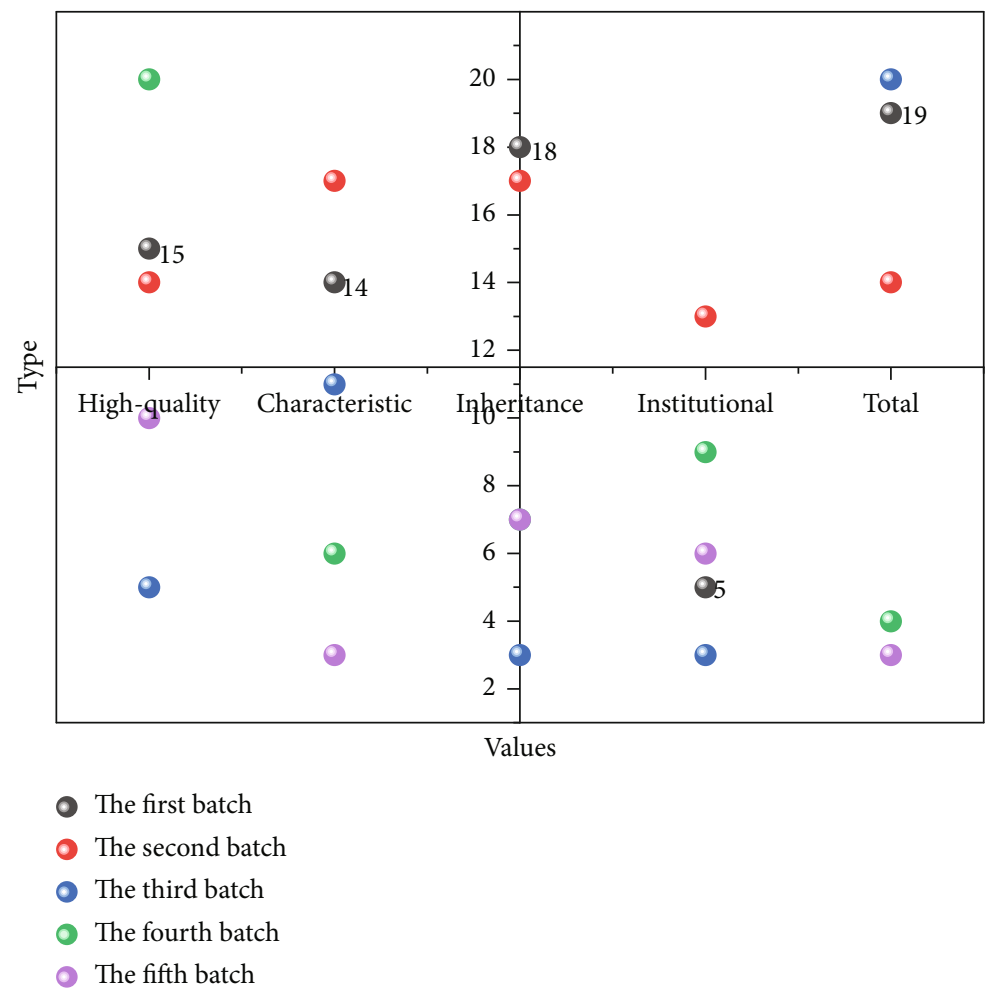

Figure 7: Classification of types of public cultural service system demonstration projects.

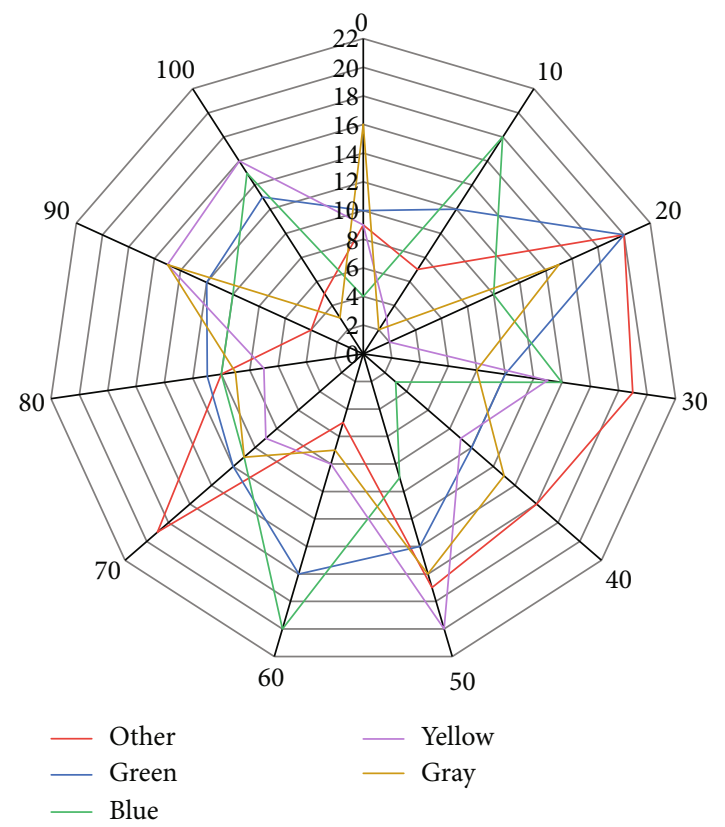

Figure 8: Test results of the intelligent government control system.

financing project. After the financing process is completed, the effect of financing will be summarized and analyzed, and the efficiency and effectiveness of the use of the financed funds will be evaluated comprehensively by combining theoretical analysis and data analysis, the projects with high efficiency in the use of financing funds will continue to be maintained and improved in future financing, and the deficiencies found will be rectified in time, to realize the effective management and efficient use of financing funds.

\section{Conclusion}

Modular switchgear can be suitable for complex and more demanding power distribution places. The digital terminal part uses advanced microelectronics and Internet of things technology, and the design idea is an advanced modular idea, which makes the power switchgear combined with new electronic technology and can realize intelligent control. This study mainly focuses on the construction of the business framework and human resource management framework for the instigation and financing of local government investment and financing platform, and no specific countermeasures are formulated for other aspects that affect the financing risks of local government investment and financing platform, which is the deficiency and limitation of this researcher. In the future, we will deepen the study and research on financing risk control and continue to pay attention to the financing of local government investment and financing platforms. Analyze the shortcomings of the traditional fixed-length data item device communication protocol in MPO-DM, and design a dual middleware unified communication protocol based on variable-length data items, so that the end-side middleware can realize the efficient conversion of device protocols and multiple PaaS platform protocols and enhance the robustness of the system to cope with PaaS protocol changes. The test results show that the DMUP protocol conversion is low in complexity and high in 
communication efficiency. The system is designed in detail from three aspects: system architecture, system functionality, and network topology, and specific implementations are given for end-side middleware and application-side middleware. Through the functional demonstration and performance test of the actual system, it shows that the system can interact with the PaaS platform and improve the level of data visualization and user software convenience and has good multi-PaaS access and data response performance, which effectively verifies the feasibility and effectiveness of the MPO-DM model.

\section{Data Availability}

The data used to support the findings of this study are available from the corresponding author upon request.

\section{Conflicts of Interest}

The author declares that he has no known competing financial interests or personal relationships that could have appeared to influence the work reported in this paper.

\section{References}

[1] A. Akhunzada, S. Islam, and S. Zeadally, "Securing cyberspace of future smart cities with $5 \mathrm{G}$ technologies," IEEE Network, vol. 34, no. 4, pp. 336-342, 2020.

[2] Z. Salih Ageed, R. M. S. Zeebaree, M. Mohammed Sadeeq et al., "A survey of data mining implementation in smart city applications," Qubahan Academic Journal, vol. 1, no. 2, pp. 91-99, 2021.

[3] B. Qin and S. Qi, "Digital transformation of urban governance in China: the emergence and evolution of smart cities," Digital Law Journal, vol. 2, no. 1, pp. 29-47, 2021.

[4] F. Sartipi, "Dynamic data processing for building energy consumption," Journal of Construction Materials, vol. 2, pp. 2-4, 2021.

[5] S. K. Rao and R. Prasad, "Impact of 5G technologies on smart city implementation," Wireless Personal Communications, vol. 100, no. 1, pp. 161-176, 2018.

[6] S. Chatterjee, A. K. Kar, and M. P. Gupta, "Critical success factors to establish $5 \mathrm{G}$ network in smart cities," Journal of Global Information Management, vol. 25, no. 2, pp. 15-37, 2017.

[7] S. M. Bohloul, "Smart cities: a survey on new developments, trends, and opportunities," Journal of Industrial Integration and Management, vol. 5, no. 3, pp. 311-326, 2020.

[8] C. Feng, K. Yu, A. K. Bashir et al., "Efficient and secure data sharing for $5 \mathrm{G}$ flying drones: a blockchain-enabled approach," IEEE Network, vol. 35, no. 1, pp. 130-137, 2021.

[9] G. Tucker, "Sustainable product lifecycle management, industrial big data, and Internet of things sensing networks in cyberphysical system-based smart factories," Journal of SelfGovernance and Management Economics, vol. 9, no. 1, pp. 919, 2021.

[10] X. Chen, "The development trend and practical innovation of smart cities under the integration of new technologies," Frontiers of Engineering Management, vol. 6, no. 4, pp. 485-502, 2019.
[11] L. Belli, A. Cilfone, L. Davoli et al., "IoT-enabled smart sustainable cities: challenges and approaches," Smart Cities, vol. 3, no. 3, pp. 1039-1071, 2020.

[12] O. B. Mora-Sanchez, E. Lopez-Neri, E. J. Cedillo-Elias, E. Aceves-Martinez, and V. M. Larios, "Validation of IoT infrastructure for the construction of smart cities solutions on living lab platform," IEEE Transactions on Engineering Management, vol. 68, no. 3, pp. 899-908, 2021.

[13] L. D. Radu, "Disruptive technologies in smart cities: a survey on current trends and challenges," Smart Cities, vol. 3, no. 3, pp. 1022-1038, 2020.

[14] J. Zhang, Y. Wang, S. Li, and S. Shi, "An architecture for IoTenabled smart transportation security system: a geospatial approach," IEEE Internet of Things Journal, vol. 8, no. 8, pp. 6205-6213, 2021.

[15] B. Ai, A. F. Molisch, M. Rupp, and Z. D. Zhong, "5G key technologies for smart railways," Proceedings of the IEEE, vol. 108, no. 6, pp. 856-893, 2020.

[16] L. Chettri and R. Bera, "A comprehensive survey on Internet of things (IoT) toward 5G wireless systems," IEEE Internet of Things Journal, vol. 7, no. 1, pp. 16-32, 2020.

[17] M. S. Răboacă, N. Bizon, and P. Thounthong, "Intelligent charging station in $5 \mathrm{G}$ environments: challenges and perspectives," International Journal of Energy Research, vol. 45, no. 11, pp. 16418-16435, 2021.

[18] C. Zhang, M. Dong, and K. Ota, "Fine-grained management in 5G: DQL based intelligent resource allocation for network function virtualization in C-RAN," IEEE Transactions on Cognitive Communications and Networking, vol. 6, no. 2, pp. 428435, 2020.

[19] H. Yang, Z. Sun, G. Jiang, F. Zhao, X. Lu, and X. Mei, “Cloudmanufacturing-based condition monitoring platform with $5 \mathrm{G}$ and standard information model," IEEE Internet of Things Journal, vol. 8, no. 8, pp. 6940-6948, 2021.

[20] Y. Shi, Q. Han, W. Shen, and H. Zhang, "Potential applications of $5 \mathrm{G}$ communication technologies in collaborative intelligent manufacturing," IET Collaborative Intelligent Manufacturing, vol. 1, no. 4, pp. 109-116, 2019. 